Background and Aim Serum bilirubin measurement is still the cornerstone method to assess hyperbilirubinemia in NN this method is invasive, painful, costly, needs more than one prick and may need to anemia due to repeated blood sampling.

Aim To compare trancutaneous bilirubin measurment with serum bilirubin.

Patients and Methods An average of three readings at different sites on the skin of NN using the bilirubinometer was taken babies included were FT, with indirect hyperbilirubinaemia, not exposed to phototherapy. Blood samples were collected at the same time for comparison.

Results A total of 101 measurements for each method were performed 67 babies were $>2.5 \mathrm{~kg}, 34$ babies were $<2.5 \mathrm{~kg}$. Age range was few hrs to 14 days.

There was excellent matching between the two methods of bilirubin measurement regardless of gestational age, birth weight, sex, post natal age.

Conclusion Transcutaneous bilirubin measurement is an alternative reliable way to assess hyperbilirubinaemia that avoids the drawbacks of blood sampling It is highly recommended in OPD, ER, NNU.

\section{ASSOCIATION OF TRANSCUTANEOUS CARBON MONOXIDE AND BILIRUBIN LEVELS IN HEALTHY TERM NEWBORNS}

doi:10.1136/archdischild-2012-302724.1330

D Gonulal, B Bilgin, 0 Altun Koroglu, M Yalaz, N Kultursay. Neonatology, Ege University Faculty of Medicine, Izmir, Turkey

Background and Aims End tidal measurement of carbonmonoxide (CO) as a side product of hemoglobin turn over may be an indicator of bilirubin production and hemolysis. We aimed to evaluate the association of transcutaneus $\mathrm{CO}$ measurement and bilirubin levels in healthy term newborns as an alternative to end tidal CO.

Methods A total number of 390 infants were recruited in the study. Infants who were born in our hospital $(n=340)$ were followed by daily transcutaneus $\mathrm{CO}$ and capillary bilirubin measurements starting from birth to discharge. Remaning 50 infants were admitted for hyperbilirubinemia treatment after 72 hours of life. Transcutaneous CO measurements were done by Masimo pulse oxymeter. Results Capillary bilirubin and transcutaneus CO levels on 24 and 48 hours of life were positively correlated (Spearman correlation coefficients $r=0.21$ and 0.87 respectively, $p$ values for both analyses $<0.001)$. Bilirubin levels and transcutaneous CO measurements were levels were also positively correlated in infants admitted for hyperbilirubinemia (Spearman correlation coefficient $r=0.41$, $p<0.001$ ).

Conclusion Our data showed positive and statistically significant correlations between bilirubin and transcutaneous $\mathrm{CO}$ measurements not in only healthy term newborns but also in newborns with hyperbilirubinemia. Therefore transcutaneous $\mathrm{CO}$ measurement deserves attention in order to obtain threshold levels for prediction of severe hyperbilirubinemia.

\section{PERCUTANEOUS TRANSHEPATIC ULTRASOUND-GUIDED CARDIAC CATHETERIZATION IN A FETAL LAMB MODEL}

doi:10.1136/archdischild-2012-302724.1331

${ }^{1,2} \mathrm{~A}$ Edwards, ${ }^{3} \mathrm{~S}$ Menahem, ${ }^{1}$ I Nitsos, ${ }^{4} Y$ Chan, ${ }^{1} \mathrm{~A}$ Veldman, ${ }^{5} \mathrm{D}$ Schranz, ${ }^{1} \mathrm{~F}$ Wong. ${ }^{1}$ The Ritchie Centre, Monash University; ${ }^{2}$ Perinatal Services, ${ }^{3}$ Fetal Cardiac Unit; ${ }^{4}$ Department of Pathology, Monash Medical Centre, Melbourne, VIC, Australia; ${ }^{5}$ Department of Paediatric Cardiology, Justus Liebig University, Giessen, Germany

Background Fetal cardiac intervention may alter the progression of cardiac conditions. Fetal heart access by direct puncture of the fetal heart under ultrasound guidance has been established, but with considerable technical difficulties. We aim to investigate the feasibility of fetal cardiac access using a percutaneous transhepatic approach in the mid-gestational fetal lamb, as a model for human fetus.

Method Eight fetal lambs of 95-97 days gestation (term 147 days) were studied. Under ultrasound guidance, the fetal right hepatic vein was punctured percutaneously, using a 16GA intravenous cannula (BD Angiocath). A coronary catheter (FineCross ${ }^{\mathrm{TM}} \mathrm{MG}$, Terumo) was inserted into the 16 GA cannula, over a 0.014inch guidewire, and advanced into the inferior vena cava and the right atrium. Contrast was injected to document position of the catheter. Three fetal lambs were to be euthanized at the end of the procedure to evaluate blood loss. The rest were to deliver vaginally at term, and euthanized for postmortem examination.

Results Percutaneous fetal cardiac access was successful in 7 out of the 8 fetuses. All 4 heart chambers were catheterized in the last 3 fetuses. One fetus died during the procedure, post-mortem showed pericardial and peritoneal haemorrhage. The other two fetuses dedicated for immediate post-mortem, after having survived the procedure, had small haemoperitoneum. Averaged fetal weight was $1027 \pm 153$ g. All other lambs were born normally at term.

Conclusion Ultrasound-guided fetal cardiac catheterization through a percutaneous transhepatic approach is feasible. Our experience provides a potentially safer route for human fetal cardiac intervention at midgestational age

\section{PREVALENCE OF ENTERIC COLONIZATION WITH VANCOMYCIN-RESISTANT ENTEROCOCCI IN INTENSIVE CARE UNITS IN SHIRAZ, IRAN}

doi:10.1136/archdischild-2012-302724.1332

${ }^{1} A$ Afkhamzadeh, ${ }^{2} \mathrm{~A}$ Delpisheh. ${ }^{1} K u r d i s t a n$ University of Medical Sciences, Sanandaj; 2llam University of Medical Sciences, Ilam, Iran

Background and Aims Vancomycin-resistant enterococci (VRE) are among the most feared hospital pathogens. The present nested case-control study aimed to determine the prevalence and risk factors of enteric colonization of VRE in ICUs in a tertiary hospital in Iran

Methods Serial rectal swabs were obtained every five days from all hospitalized patients (70 cases) in six ICUs and one CCU until patient's discharge. During the study 100 samples of rectal swab in sterile situation were taken. Then enterococci detection and susceptibility were done by disk diffusion and MIC dilution method.

Results A total of 9 of the 70 patients (12.85\%) were colonized with VRE. five patients who had at least a negative test for VRE - at the time of hospitalization- were colonized with resistant enterococci which demonstrated an incident rate of colonization as $7 \%$ at three months of study period. Univariate analysis showed that prior antibiotic consumption, vancomycin and third generation cephalosporins, and duration of vancomycin use were significant risk factors for VRE colonization $(p<0.05)$. There were no significant association between prevalence of VRE and variables such as age, ward, history of admission, history of antibiotic use, duration of hospitalization and underlying disease $(p>0.05)$. The logistic regression showed that the consumption of vancomycin was the independent risk factors for VRE colonization

Conclusions Prevalence of VRE in ICUs in the present study was high in consistence with national studies confirming that this organism might be endemic in tertiary large hospitals in Iran. INFECTIONS IN NEWBORN INFANTS ADMITTED TO NEONATAL INTENSIVE CARE UNIT

doi:10.1136/archdischild-2012-302724.1333 